\title{
Photorealistic Visualization of Fluorescence Materials with Dual Surface Scattering
}

\author{
D.D. Zhdanov ${ }^{1}$, I.S. Potemin ${ }^{1}$, A.D. Zhdanov ${ }^{1}$, V.G. Sokolov², S.V. Ershov ${ }^{2}$, E.U. Denisov ${ }^{2}$ \\ ddzhdanov@mail.ru|ipotemin@yandex.ru|adzhdanov@itmo.ru|sokolovv@gmail.com|sergey_65@mail.ru \\ leed@spp.keldysh.ru \\ ${ }^{1}$ ITMO University, Saint Petersburg, Russia; \\ ${ }^{2}$ Keldysh Institute of Applied Mathematics RAS, Moscow, Russia.
}

We describe a simple method to extract fluorescent characteristics of a surface by combining measurements by a "usual" gonioreflectormeter GSCM-4 and fluorimeter FP-8600. The fluorescent BDF consists of three components: glossy near-specular peak which is not fluorescent and white, highly diffuse "passive" part which is also not fluorescent but colored, and fluorescent part. The latter obviates Kasha's-Vavilov's rule (factorization) with good accuracy. The BDFs obtained were used in rendering and shown good visual match with the natural photographs.

Keywords: fluorescence, fluorescent emission, fluorescence efficiency, Bi-directional Scattering Function (BSDF)

\section{Introduction}

Usually light scattered by a surface or a turbid medium illuminated by a monochrome light has the same wavelength, as the incident one. This is however not always; the effect when scattered light has another wavelength is named fluorescence.

Fluorescence occurs at the molecular level. Roughly 11, an incident photon while interacting with a molecule, kicks it into an excited state and this photon "disappears" instead of being elastically scattered or gone into heat (absorption). There can be several excited energy levels, but all of them are "reachable" for those incident wavelengths that interact with the molecule inelastically.

Then the molecule returns to the ground state emitting photon whose energy is thus also fixed: it is the difference between the ground and the excited energy level. Ideally it means a discrete spectrum of emission, but in reality because of the thermal motion and other factors, the peaks blur and emission has a continuous spectrum. Most frequently, in fluorescence a short-wave light (UV or at least blue) is converted into a visible range; so we can see them under an UV lamp in spite a human eye can not sense UV.

The bulk material that contain fluorescent molecules can be homogeneous (when it consists of that molecules entirely) or not, when there is a "passive" material and fluorescent molecules dispersed in it. In this latter case it can be a solution or particles of completely made of fluorescent material.

Fluorescent emission from molecular solution is rather isotropic. But in case of particles the situation is different. We can consider the interior of that particle as uncorrelated random source (of isotropic light). Its local amplitude is proportional to the local intensity of the incident light, diffracted in the particle. This already creates some anisotropy, and diffraction of that wave field inside particle adds more. As a result, fluorescent emission from a particle can be anisotropic. Nevertheless its angular distribution is quite smooth, without sharp peaks [2], [3].

The radiance of a "usual" surface under monochrome parallel illumination is calculated from its $\mathrm{BDF}[4] f$ as

$$
L(\boldsymbol{u}, \lambda)=f(\boldsymbol{u}, \boldsymbol{v} ; \lambda) I(\lambda)
$$

where $I$ is the spectral density of irradiance, $\boldsymbol{u}$ is direction of observation and $v$ is direction of illumination and $\lambda$ is wavelength. A fluorescent surface can be described by an extension of BDF, which now depends on two wavelengths, of illumination and of observation. Now the spectral density of radiance at wavelength $\lambda$ is

$$
L(\boldsymbol{u}, \lambda)=\int f\left(\boldsymbol{u}, \boldsymbol{v} ; \lambda, \lambda^{\prime}\right) I\left(\lambda^{\prime}\right) d \lambda^{\prime}
$$

where $I$ is also spectral density of irradiance, see [5], [6], [7], [8].

Usually besides fluorescence there is also a "passive" scattering when the light is re-emitted at the same wavelength, so $\mathrm{BDF}$ is

$$
f\left(\boldsymbol{u}, \boldsymbol{v} ; \lambda, \lambda^{\prime}\right)=f^{(f)}\left(\boldsymbol{u}, \boldsymbol{v} ; \lambda, \lambda^{\prime}\right)+f^{(p)}(\boldsymbol{u}, \boldsymbol{v} ; \lambda) \delta\left(\lambda-\lambda^{\prime}\right)
$$

where $f^{(f)}$ is the pure fluorescent part (continuous in both wavelengths) and $f^{(p)}$ is the passive part.

From the quantum nature of the fluorescent effect it follows that frequently at the molecular level the spectrum of emission is independent from the incident wavelength, which is termed Kasha-Vavilov rule [9], [10]. If so, this will also hold for a bulk material, and then the fluorescent component of BDF factors as done in [6], [7]:

$$
f^{(f)}\left(\boldsymbol{u}, \boldsymbol{v} ; \lambda_{\text {out }}, \lambda_{\text {in }}\right)=E\left(\boldsymbol{u}, \boldsymbol{v} ; \lambda_{\text {out }}\right) A\left(\boldsymbol{v} ; \lambda_{\text {in }}\right)
$$

Here $E$ is termed emission and $A$ is termed excitation, or, sometimes, quantum yield (for the latter we must also use the scale by the ratio of frequencies of the incident and emitted photons). Emission spectrum is normalized so that

$$
\int E\left(\boldsymbol{u}, \boldsymbol{v} ; \lambda_{\text {out }}\right) d \lambda_{\text {out }}=1
$$

The above factorization is not the general rule and it violates in some cases [9], [11].

\section{Acquisition of fluorescent BDF}

Like a usual BDF, it can be either measured or calculated. The latter requires that we know all detailed physical properties of all substances (passive and fluorescent) involved, geometry and distribution of size, position and shape of particles and so on. Then we simulate light interaction with that material assuming parallel monochrome illumination. Usually one must account for diffraction (see above), and this requires wave optics. Although this way is possible, but it is rare that all the data are known at the necessary detail.

Or one can measure this BDF, but since it depends on two wavelengths, of illumination and of observation, it can not be measured on such devices like GSCM-4 used to measure "usual" BDFs. In the latter case it is enough to use one monochromator, in either illumination or observation channel. For a fluorescent BDFs we need $t w o$, in both channels, see Figure 1.

In principle it is possible to take a device like GSCM-4 and place additional monochromator in the illumination channel. This 
device would measure dependence on illumination wavelength, observation wavelength, illumination direction and observation direction. The authors of [5] just followed that way and assembled a reduced version of that device which operates in the plane of incidence.

We did not have a possibility for an optical device manufacturing and decided to use a ready fluorimeter available on the market instead. Regrettably most of them do not measure angular dependence.

We have access to FP-8600 manufactured by JASCO. Besides it also measures only one combination $(\boldsymbol{u}, \boldsymbol{v})$, there is yet another problem with this device. It does not output the ready-to-use values of $\mathrm{BDF}$ at least for a single illumination/observation condition. Its output is in such units that one needs do some calibration of the device and postprocessing of data to get the necessary values.

This approach is more accurate and detailed than the one used in [6], [7] whose authors used an RGB measuring camera and a set of varying polychrome illuminations. The use of a polychrome illumination instead of a monochrome one only required a more complex processing procedure because mathematically an acquisition of a linear operator requires measuring of its action on a sufficient number of different input vectors. Using vectors with only one not zero component (monochrome illumination) is more straightforward and no more. But the use of an RGB camera makes it impossible to measure the spectrum of emission.

\section{Samples}

For this experiment we used two fluorescent samples of thin paper-like opaque sheets. Although GSCM-4 can not measure fluorescent BDFs, it still can measure the angular dependence, though the result is some mixture in wavelengths and also it has wrong scale (its total reflection may exceed 100\%). Although these measurements were helpful. They shown the angular distribution of scattered light consists of two parts. One is a sharp near-specular peak, which comes from reflection of the rather glossy front surface. It is not fluorescent because fluorescent emission has a rather smooth angular distribution. The second component is, on the contrary, close to Lambert.

The gloss peak is nearly not affected by the smooth fluorescent emission, so its measurement by GSCM-4 is reliable. We used this part, zeroing the off-specular area.

As to the off-specular part, we assume it is Lambert. As to the wavelength dependence, it was calculated from measurements by FP-8600. Below we shall explain how we did that.

\section{What happens in FP-8600}

In FP-8600, the sample is illuminated by nearly parallel light at $\sigma=30^{\circ}$. This light passes the first monochromator which leaves only a narrow spectral interval. The detector collects light in a narrow cone about observation direction at $\gamma=60^{\circ}$ and this light passes the second monochromator, see Figure 1.

Since observation is in the off-specular area and BRDF is rather smooth, we can forget angular distribution of illumination and angular distribution the sensor sensitivity and assume illumination is parallel and observation too.

Spectral density of radiance of light reflected by the sample in direction $\boldsymbol{u}$ at wavelength is therefore

$$
L_{\text {out }}\left(\boldsymbol{u}, \lambda ; \lambda_{\text {in }}\right)=\int f\left(\boldsymbol{u}, \boldsymbol{v} ; \lambda, \lambda^{\prime}\right) F_{1}\left(\lambda^{\prime}, \lambda_{\text {in }}\right) I\left(\lambda_{\text {in }}\right) d \lambda^{\prime}
$$

Here $\lambda_{\text {in }}$ is wavelength set in the $1^{\text {st }}$ monochromator (for illumination), $F_{1}$ is transmission of that monochromator, $\lambda^{\prime}$ is wavelength of illumination, which due to final bandpass of the filter spreads over some interval near $\lambda_{\text {in }}$.

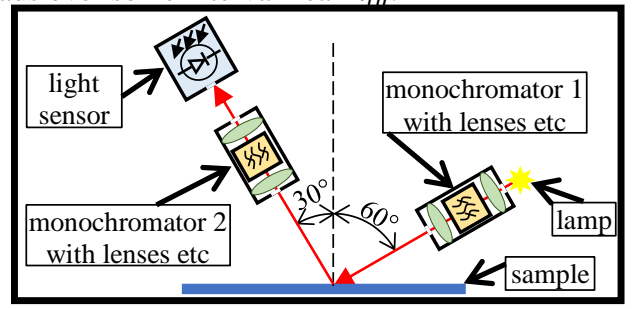

Figure 1. Scheme of the device FP-8600

Specification of the device does not say it clearly what it outputs, but because of presence of the word "intensity" in the output file one can assume it is the spectral density of the power flow of reflected light, up to a constant scale. That is, the records in the output file are

$$
R\left(\lambda_{\text {out }} ; \lambda_{\text {in }}\right)=C\left(\lambda_{\text {out }}\right) L_{\text {out }}\left(\boldsymbol{u}, \lambda_{\text {out }} \mid \lambda_{\text {in }}\right)
$$

where $\lambda_{\text {out }}$ is wavelength set in the $2^{\text {nd }}$ monochromator (for observation) and $c$ is that scale factor, which in principle can depend on wavelength. Cross-sections for just three incident wavelength are shown in Figure 2.

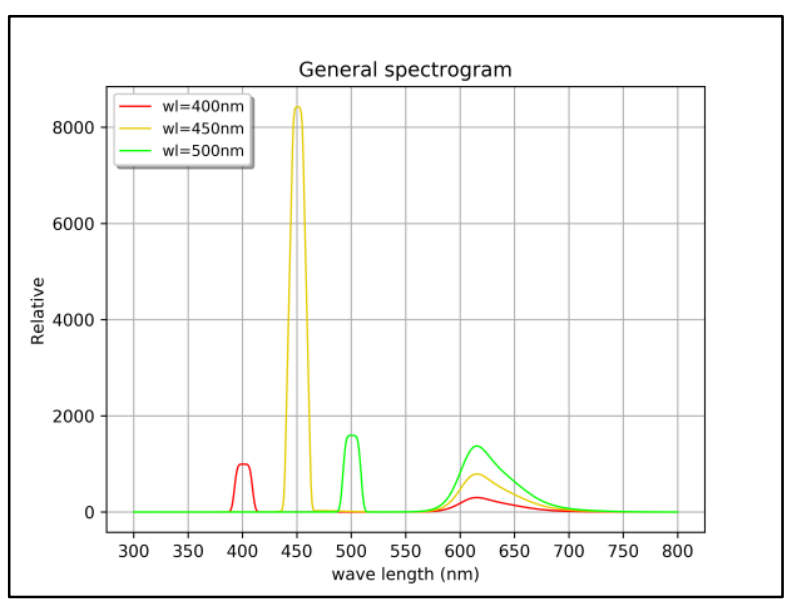

Figure 2. Raw output spectrogram of fluorescent "sample1" for just three wavelengths of illumination: 400,450 and $500 \mathrm{~nm}$.

$$
\begin{aligned}
& \text { Combining, we have } \\
& R\left(\lambda_{\text {out }}\right)=C\left(\lambda_{\text {out }}\right) \int f\left(\boldsymbol{u}, \boldsymbol{v} ; \lambda_{\text {out }}, \lambda^{\prime}\right) F_{1}\left(\lambda^{\prime}, \lambda_{\text {in }}\right) I\left(\lambda_{\text {in }}\right) d \lambda^{\prime}
\end{aligned}
$$

Applying decomposition (2),

$$
\begin{aligned}
R\left(\lambda_{\text {out }} ; \lambda_{\text {in }}\right) & =C\left(\lambda_{\text {out }}\right) I\left(\lambda_{\text {in }}\right) \int f^{(f)}\left(\boldsymbol{u}, \boldsymbol{v} ; \lambda_{\text {out }}, \lambda^{\prime}\right) F_{1}\left(\lambda^{\prime}, \lambda_{\text {in }}\right) d \lambda^{\prime} \\
& +C\left(\lambda_{\text {out }}\right) f^{(p)}\left(\boldsymbol{u}, \boldsymbol{v} ; \lambda_{\text {in }}\right) F_{1}\left(\lambda_{\text {out }}, \lambda_{\text {in }}\right) I\left(\lambda_{\text {in }}\right)
\end{aligned}
$$

For a good monochromator, $F_{1} \neq 0$ only in a narrow interval, while $f^{(f)}$ depends on wavelengths smoothly. So

$$
\begin{aligned}
R\left(\lambda_{\text {out }} ; \lambda_{\text {in }}\right) & =C\left(\lambda_{\text {out }}\right) I\left(\lambda_{\text {in }}\right) f^{(f)}\left(\boldsymbol{u}, \boldsymbol{v} ; \lambda_{\text {out }}, \lambda_{\text {in }}\right) \bar{F}_{1}\left(\lambda_{\text {in }}\right) \\
& +C\left(\lambda_{\text {out }}\right) f^{(p)}\left(\boldsymbol{u}, \boldsymbol{v} ; \lambda_{\text {in }}\right) F_{1}\left(\lambda_{\text {out }}, \lambda_{\text {in }}\right) I\left(\lambda_{\text {in }}\right)
\end{aligned}
$$

where

$$
\bar{F}_{1}\left(\lambda_{\text {in }}\right) \equiv \int F_{1}\left(\lambda^{\prime}, \lambda_{\text {in }}\right) d \lambda^{\prime}
$$

Since $C, F_{1}$ and $I$ are unknown, we need some "calibration" to get the BDF $f^{(p)}, f^{(f)}$ from the measurement results. To this end, we used measurement of a diffuse etalon w/o fluorescence, but with 
known $\mathrm{BDF}^{1}$. Its cross-sections for just three incident wavelength are shown in Figure 3. So, for the passive etalon the above equation yields

$$
R_{e}\left(\lambda_{\text {out }} ; \lambda_{\text {in }}\right)=C\left(\lambda_{\text {out }}\right) f_{e}\left(\boldsymbol{u}, \boldsymbol{v} ; \lambda_{\text {in }}\right) F_{1}\left(\lambda_{\text {out }}, \lambda_{\text {in }}\right) I
$$

\section{Processing of data}

To begin with, one can see that (5) consists of two components. The first which comes from passive scattering, is nearly singular, i.e. it is sharp peak near the diagonal $\lambda_{\text {out }}=\lambda_{\text {in }}$. The second is smooth, see Figure 4, and near diagonal the values of the first component are much higher. Similarly, (6) is also a sharp diagonal peak.

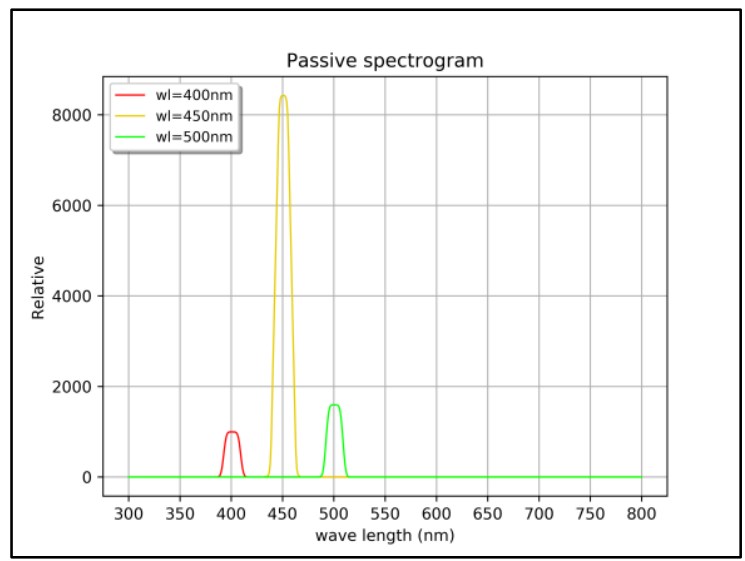

Figure 3. Raw output spectrogram of etalon sample for just three wavelengths of illumination: 400,450 and $500 \mathrm{~nm}$.

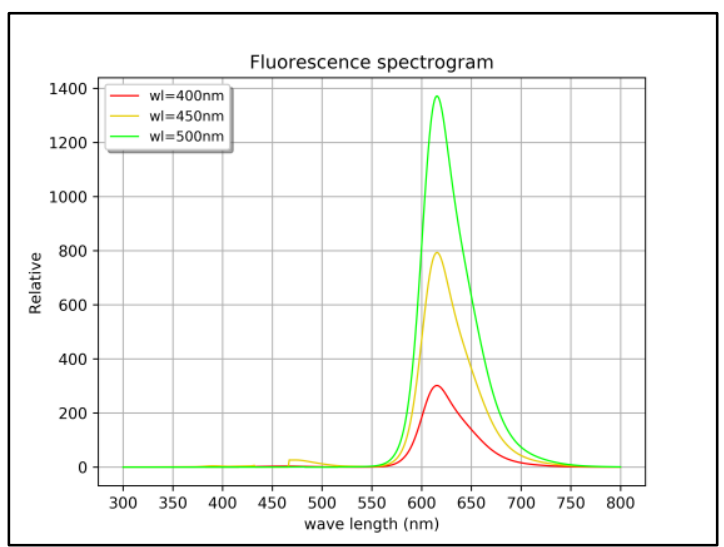

Figure 4. Raw output spectrogram of fluorescent "sample1" after zeroing the near-diagonal components, shown for just three wavelengths of illumination: 400,450 and $500 \mathrm{~nm}$.

So, if we integrate over a narrow spectral interval around $\lambda_{\text {in }}$ assuming the sensor sensitivity etc. smoothly depend on wavelength so $C(\lambda)$ does not vary much over $\left[\lambda_{\text {in }}-\epsilon, \lambda_{\text {in }}+\epsilon\right]$, then

$$
\int_{\lambda_{\text {in }}-\epsilon}^{\lambda_{\text {in }}+\epsilon} R\left(\lambda_{\text {out }} ; \lambda_{\text {in }}\right) d \lambda_{\text {out }} \approx C\left(\lambda_{\text {out }}\right) f^{(p)}\left(\boldsymbol{u}, \boldsymbol{v} ; \lambda_{\text {in }}\right) \bar{F}_{1}\left(\lambda_{\text {in }}\right) I\left(\lambda_{\text {in }}\right)
$$

$\int_{\lambda_{\text {in }}-\epsilon}^{\lambda_{\text {in }}+\epsilon} R_{e}\left(\lambda_{\text {out }} ; \lambda_{\text {in }}\right) d \lambda_{\text {out }}=C\left(\lambda_{\text {out }}\right) f_{e}\left(\boldsymbol{u}, \boldsymbol{v} ; \lambda_{\text {in }}\right) \bar{F}_{1}\left(\lambda_{\text {in }}\right) I\left(\lambda_{\text {in }}\right)$

from what it follows that

$$
f^{(p)}\left(\boldsymbol{u}, \boldsymbol{v} ; \lambda_{i n}\right) \approx \frac{f_{e}\left(\boldsymbol{u}, \boldsymbol{v} ; \lambda_{i n}\right)}{\bar{R}_{e}\left(\lambda_{i n}\right)}
$$

while away from diagonal (5) yields

$$
f^{(f)}\left(\boldsymbol{u}, \boldsymbol{v} ; \lambda_{\text {out }}, \lambda_{\text {in }}\right)=\frac{R\left(\lambda_{\text {out }} ; \lambda_{\text {in }}\right)}{\bar{R}_{e}\left(\lambda_{\text {in }}\right)}, \quad\left|\lambda_{\text {out }}-\lambda_{\text {in }}\right|>\epsilon
$$

The near diagonal values of $f^{(f)}\left(\boldsymbol{u}, \boldsymbol{v} ; \lambda_{\text {out }}, \lambda_{\text {in }}\right)$ are unknown, but this function is smooth and we can interpolate them.

Spectrograms of the passive part obtained this way are shown in Figure 5.

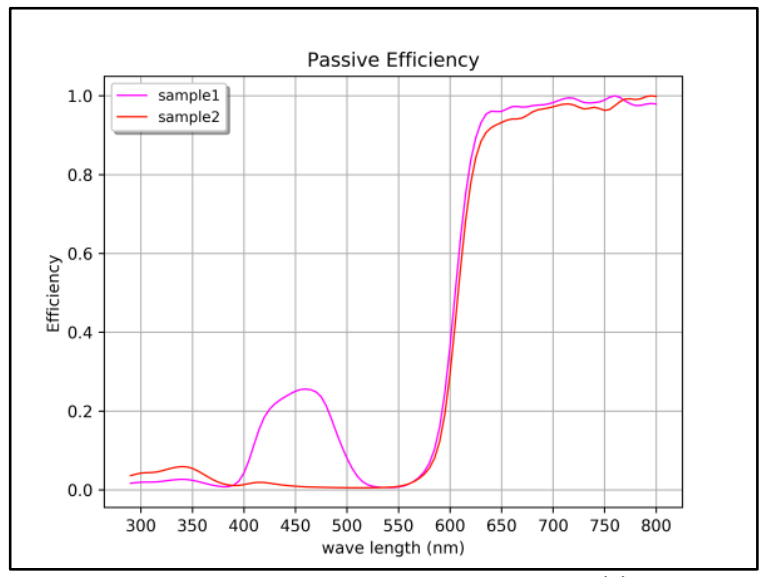

Figure 5. Spectrograms of the passive part $f^{(p)}\left(\boldsymbol{u}, \boldsymbol{v} ; \lambda_{\text {in }}\right)$

\section{Factorization of the fluorescent BDF}

Applying factorization (3) to (8) we have

$$
\begin{gathered}
A\left(\boldsymbol{v} ; \lambda_{\text {in }}\right)=\int_{\left|\lambda_{\text {out }}-\lambda_{\text {in }}\right|>\epsilon} \frac{R\left(\lambda_{\text {out }} ; \lambda_{\text {in }}\right)}{\bar{R}_{e}\left(\lambda_{\text {in }}\right)} d \lambda_{\text {out }} \\
E\left(\boldsymbol{u}, \boldsymbol{v} ; \lambda_{\text {out }}\right)=\int^{\frac{R\left(\lambda_{\text {out }} \lambda_{\text {in }}\right)}{\bar{R}_{e}\left(\lambda_{\text {in }}\right)}} \frac{\lambda_{\text {in }}}{A\left(\boldsymbol{v} ; \lambda_{\text {in }}\right)} d \lambda_{\text {in }},\left|\lambda_{\text {out }}-\lambda_{\text {in }}\right|>\epsilon
\end{gathered}
$$

Notice that usually emission is zero in the wavelength range where $A>0$, so $E$ is all the same 0 in the near-diagonal area. Results of this procedure are shown in Figures 6 and 7.

As said in the end of Section 1, this factorization may violate, so it was not evident whether it can or can not be applied to other samples. It happened it can, i.e. (3) is satisfied with good accuracy. The shape of spectral emission graphs for both samples is the same, so the difference is the visible color (see Figure 8) is due to, first, excitation, and, second, passive part of BDF.

\section{Putting all together}

We therefore have all components of BDF. First we clear the off-specular value (outside of the cone $10^{\circ}$ about the specular direction) in the GSCM-4 results. This gives us the gloss peak.

Second, we take the passive part of the smooth BDF component from (7). This gives us BDF for single combination $(\boldsymbol{u}, \boldsymbol{v})$ but since (we assumed that) Lambert angular dependence, it applies to all of them.

${ }^{1}$ Obtained by measurement in GSCM-4 


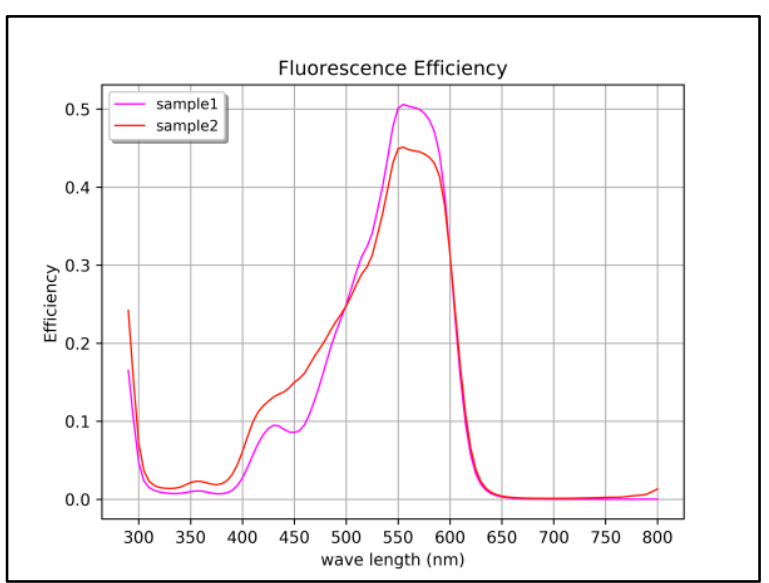

Figure 6. Spectrograms of excitation $A(\boldsymbol{v} ; \lambda)$.

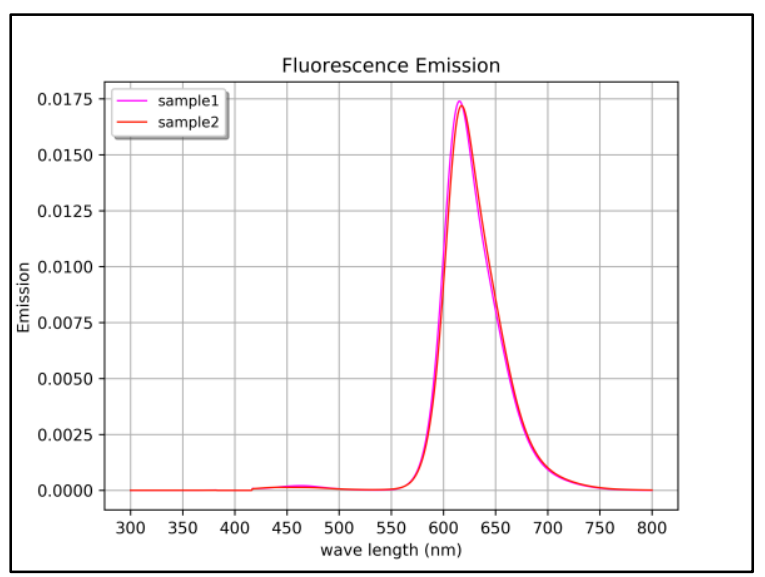

Figure 7. Spectrograms of emission $E(\boldsymbol{u}, \boldsymbol{v} ; \lambda)$.

Third, we take the fluorescent part of the smooth BDF component from (8). In fact we even used factorization described in Section 6, because this decreases various random errors because the components $E$ and $A$ are averages over one wavelength. This gives us BDF for single combination $(\boldsymbol{u}, \boldsymbol{v})$ but since (we assumed that) Lambert angular dependence, it applies to all of them.

\section{Rendering}

Once we know all components of BDF we can use it in rendering. They were compared with the natural photograph made by placing the two samples in the colour evaluation device "Judge II". Rendering was done for the scene which is the model of that setup.

The results are shown in Figure 8. One can see serious visible improvements in color reproduction after the use of the BDF obtained with our method.

The natural photo of the samples in the Judge II has been obtained by Spectroradiometer Konica Minolta CA-2000. To avoid possible influence of camera software, specific tone mapping, spectral sensitivity of CCD and so on indirect approach was chosen for images preparation. An output in XYZ chromaticity coordinates measured by Konica Minolta was converted to format supported by our optical simulation software with next conversion to RGB images. The same approach was applied to the results of rendering. So the same technique was used for transformation of XYZ data to images and any divergence which can be in results of tone mapping, gamma correction and other post-processing procedures is excluded from comparison.

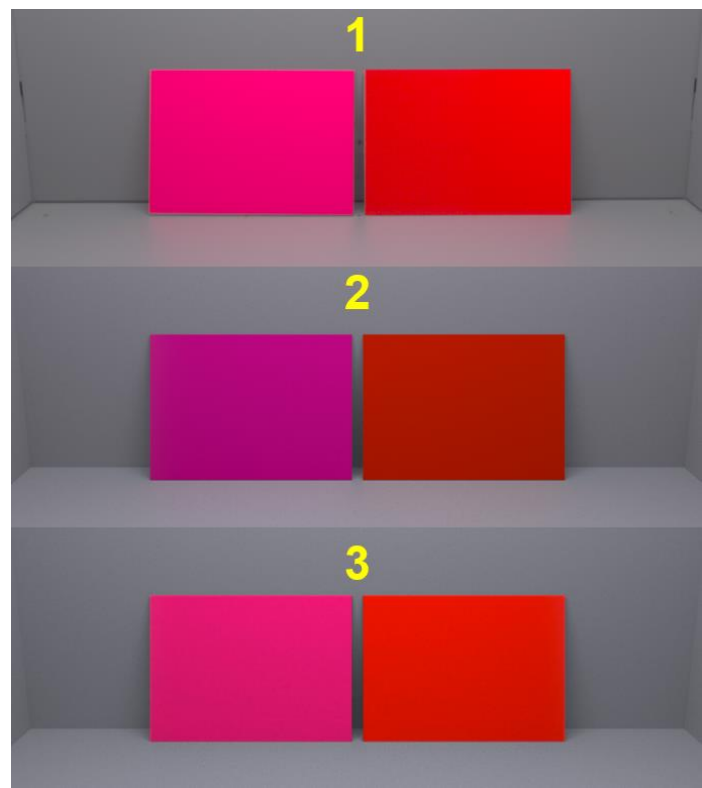

Figure 8. Top to bottom: natural photo, rendering with the BDF taken "as is" from the GSCM-4 measurements, rendering with the BDF obtained by our method.

\section{Conclusion}

As a result of the current research, we found out that the method of fluorescence support can be successfully used in 3D simulation software. It gives noticeable improvements in color reproduction of simulated objects having fluorescent properties. The main advantage of the method is its simplicity. Simple mathematical description based on diffuse reflection allows to use it in any ray tracing techniques from forward Monte-Carlo ray tracing up to bidirectional ray tracing technique with combination of forward and backward ray tracing, using photon maps etc. More significant advantage is simplicity of measuring technique which can be applied for fluorescent materials. It is combination of measurements of BSDF and usual spectrograms which can be executed with well-known measuring devices available in market.

\section{Appendix. Fluorescent BDF in MCRT}

In MCRT, after a ray hits a surface, we first choose at random its new direction (after scattering), and then, knowing the direction, the color of the new ray is calculated deterministically because it is a unique function of direction and illumination color.

In many variants of MCRT, rays have constant (unit) energy and absorption is simulated by killing rays at random with "Russian Roulette". Since rays scattered by BDF have all unit energy, their angular density equals (up to a constant scale) the angular density of scattered energy.

So for a non-fluorescent BDF the probability of ray killing is

the angular density is

$$
P=1-\int f^{(p)}(\boldsymbol{u}, \boldsymbol{v} ; \lambda) E_{\text {in }}(\lambda) d \lambda d^{2} \boldsymbol{v}
$$

$$
p(\boldsymbol{v})=\frac{\int f^{(p)}(\boldsymbol{u}, \boldsymbol{v} ; \lambda) E_{\text {in }}(\lambda) d \lambda}{\int f^{(p)}(\boldsymbol{u}, \boldsymbol{v} ; \lambda) E_{\text {in }}(\lambda) d \lambda d^{2} \boldsymbol{v}}
$$


and the spectrogram of the scattered ray is

$$
E_{\text {out }}(\lambda)=\frac{f^{(p)}(\boldsymbol{u}, \boldsymbol{v} ; \lambda) E_{\text {in }}(\lambda)}{\int f^{(p)}(\boldsymbol{u}, \boldsymbol{v} ; \lambda) E_{\text {in }}(\lambda) d \lambda}
$$

where $E_{\text {in }}(\lambda)$ and $E_{\text {out }}(\lambda)$ are spectra of the incident and scattered rays.

For fluorescent BDF, the angular density and the probability of ray killing are given by the same expressions if substitute instead of $f^{(p)}(\boldsymbol{u}, \boldsymbol{v} ; \lambda)$ we substitute the integral over wavelength of emission:

$$
\int f^{(f)}\left(\boldsymbol{u}, \boldsymbol{v} ; \lambda^{\prime \prime}, \lambda\right) d \lambda^{\prime \prime}
$$

Spectrogram of the outgoing ray is

$$
E_{\text {out }}(\lambda)=\frac{\int f^{(f)}\left(\boldsymbol{u}, \boldsymbol{v} ; \lambda, \lambda^{\prime}\right) E_{\text {in }}\left(\lambda^{\prime}\right) d \lambda^{\prime}}{\int\left(\int f^{(f)}\left(\boldsymbol{u}, \boldsymbol{v} ; \lambda, \lambda^{\prime}\right) E_{\text {in }}\left(\lambda^{\prime}\right) d \lambda^{\prime}\right) d \lambda}
$$

Therefore, adaptation of an FMCRT code to handle fluorescent surfaces is rather simple. Another situation is with BMCRT. Here, the camera ray does not represent a real physical entity.

For a non-fluorescent BDF, again, we first choose ray direction at random and then calculate its color deterministically, as a product of the incident ray color ad BDF. Angular density of direction is, by analogy with FMCRT, chosen as

$$
p(\boldsymbol{u})=\frac{\int f^{(p)}(\boldsymbol{u}, \boldsymbol{v} ; \lambda) E_{\text {in }}(\lambda) d \lambda}{\int f^{(p)}(\boldsymbol{u}, \boldsymbol{v} ; \lambda) E_{\text {in }}(\lambda) d \lambda d^{2} \boldsymbol{u}}
$$

(notice that in BMCRT $\boldsymbol{u}$ is the incident and $\boldsymbol{v}$ is the scattered ray directions).

But what to do with a fluorescent BDF? Its transformation of spectrum is matrix multiplication. So, like in BRT in crystals [12], camera ray "color" becomes a matrix. For a passive BDF it is diagonal. When camera ray is scattered by a surface, this matrix transforms as

$$
\hat{C} \mapsto \hat{C} \hat{f}(\boldsymbol{u}, \boldsymbol{v})
$$

where $\hat{f}$ is BDF "reradiance" matrix. Notice BDF is multiplied by the ray "color" from the left. Multiplication by the illumination spectrum is from the right (1), so it first interacts with this surface $\mathrm{BDF}$ and after that the color transformation matrix from that surface to camera is applied.

In BMCRT, transformation of ray "color" must take into account the number of rays, i.e. their angular density, and BMCRT ray color transforms as

$$
\hat{C} \mapsto \frac{1}{p(\boldsymbol{v} \mid \boldsymbol{u})} \hat{C} \hat{f}(\boldsymbol{u}, \boldsymbol{v})
$$

where $p(\boldsymbol{v} \mid \boldsymbol{u})$ is the angular density of scattered ray direction $\boldsymbol{v}$ when before scattering the ray has direction $\boldsymbol{u}$.

In the not fluorescent case, the density is constructed like this: it is proportional to energy (sum over spectrum) brought to the camera pixel from given scattering direction, if the scattered ray collects "white" (with constant spectrum) illumination.

In the polarized case, this can be done as well and gives

$$
p(\boldsymbol{v} \mid \boldsymbol{u})=\text { const } \times \sum_{\lambda^{\prime \prime}} C_{\lambda, \lambda^{\prime \prime}} f_{\lambda^{\prime \prime}, \lambda^{\prime}}(\boldsymbol{u}, \boldsymbol{v})
$$

where the scale factor is chosen so that $\int p(\boldsymbol{v} \mid \boldsymbol{u}) d^{2} \boldsymbol{v}=1$.

In presence of fluorescence, RGB simulation is impossible and we must operate spectral domain. Even if just 40 wavelength are used to have wavelength interval $10 \mathrm{~nm}$ (and frequently it is insufficient!), camera ray "color" is a $40 \times 40$ matrix, i.e. it contains 1600 elements. With such data size, photon maps for camera ray become hardly feasible.

And here factorization of BDF is a salvation. Indeed, now the reradiance matrix is a product of two spectrograms, emission $E$ and excitation $A$, which in Dirac's notations is written as $|E\rangle\langle A|$. Since for not fluorescent surfaces the reradiation matrix is diagonal, the camera ray color is either
- a diagonal matrix, until it hit a fluorescent surface

- a product $\left|C_{E}\right\rangle\left\langle C_{A}\right|$ after that.

In the former case, we need only $N_{\lambda}$ elements, in the latter one $2 N_{\lambda}$ elements which is still admissible.

\section{Acknowledgments}

The research was partially supported by RFBR grants No. 1901-00435 and 17-01-00363.

\section{References}

[1] Lakowicz, Joseph R. (1999). Principles of Fluorescence Spectroscopy. Kluwer Academic / Plenum Publishers. ISBN 978-0-387-31278-1

[2] P Kratohvil, J and P Lee, M and Kerker, M. Angular distribution of fluorescence from small particles. // Applied optics, vol. 17, 1978, p. 1978-1980

[3] Hang Lee, R. E. Benner, J. B. Fenn, and R. K. Chang. Angular distribution of fluorescence from liquids and monodispersed spheres by evanescent wave excitation. Appl. Opt. 18(6) 862-868 (1979)

[4] Fred E. Nicodemus. Directional Reflectance and Emissivity of an Opaque Surface. // Applied Optics, vol. 4(7), 1965, p. 767-775.

[5] Matthias B. Hullin, Johannes Hanika, Boris Ajdin, HansPeter Seidel, Jan Kautz, and Hendrik P. A. Lensch. 2010. Acquisition and analysis of bispectral bidirectional reflectance and reradiation distribution functions. In $A C M$ SIGGRAPH 2010 papers (SIGGRAPH '10), Hugues Hoppe (Ed.). ACM, New York, NY, USA, Article 97, 7 pages. DOI: https://doi.org/10.1145/1833349.1778834

[6] Ying Fu, Antony Lam, Yasuyuki Kobashi, Imari Sato, Takahiro Okabe, and Yoichi Sato. 2014. Reflectance and Fluorescent Spectra Recovery Based on Fluorescent Chromaticity Invariance under Varying Illumination. In Proceedings of the 2014 IEEE Conference on Computer Vision and Pattern Recognition (CVPR'14). IEEE Computer Society, Washington, DC, USA, 2171-2178. DOI: https://doi.org/10.1109/CVPR.2014.278

[7] Zhang, Cherry \& Sato, Imari. (2011). Separating reflective and fluorescent components of an image. Proceedings of the IEEE Computer Society Conference on Computer Vision and Pattern Recognition. p. 185-192 https://doi.org/10.1109/CVPR.2011.5995704

[8] Wilkie, Alexander \& Weidlich, Andrea \& Larboulette, Caroline \& Purgathofer, Werner. (2006). A reflectance model for diffuse fluorescent surfaces. P. 321-331. https://doi.org/10.1145/1174429.1174484

[9] Kasha's rule. Wikipedia: https://en.wikipedia.org/wiki/Kasha\%27s_rule.

[10] IUPAC. Compendium of Chemical Terminology, 2nd ed. (the "Gold Book"). Compiled by A. D. McNaught and A. Wilkinson. Blackwell Scientific Publications, Oxford (1997). Online version (2019-) created by S. J. Chalk. ISBN 09678550-9-8 https://doi.org/10.1351/goldbook

[11] del Valle, Juan Carlos and Catalán, Javier. Kasha's rule: a reappraisal. // Phys. Chem. Chem. Phys., vol. 21, no 19, 2019, p. 10061-10069. http://dx.doi.org/10.1039/C9CP00739C.

[12] Dmitry Zhdanov, Sergey Ershov, Leo Shapiro, Vadim Sokolov, Alexey Voloboy, Vladimir Galaktionov, Igor Potemin. Realistic rendering of scenes with anisotropic media // Optical Engineering, 58(8), 082413 (2019) https://doi.org/10.1117/1.OE.58.8.082413 